\title{
The Development of an Instrument for Assessing Individual Ethical Decision- making in Project-based Design Teams: Integrating Quantitative and Quali- tative Methods
}

\section{Qin Zhu, Purdue University}

Qin Zhu is a PhD student in the School of Engineering Education at Purdue University. His main research interests include global/comparative/international engineering education, engineering education policy, and engineering ethics. He received his BS degree in material sciences and engineering and first $\mathrm{PhD}$ degree in philosophy of science and technology (engineering ethics) both from Dalian University of Technology, China. His first PhD dissertation on improving the practical effectiveness of engineering ethics that draws on theories in hermeneutics, practical philosophy, and discourse ethics has recently been awarded the "Outstanding Dissertation Award" in Liaoning Province, China.

\section{Dr. Carla B. Zoltowski, Purdue University, West Lafayette}

Carla B. Zoltowski, Ph.D., is Co-Director of the EPICS Program at Purdue University. She received her B.S. and M.S. in electrical engineering and Ph.D. in engineering education, all from Purdue University. She has served as a lecturer in Purdue's School of Electrical and Computer Engineering. Dr. Zoltowski's academic and research interests include human-centered design learning and assessment, service-learning, ethical reasoning development and assessment, leadership, and assistive technology.

\section{Megan Kenny Feister, Purdue University- West Lafayette}

Megan K. Feister is a doctoral candidate in the Brian Lamb School of Communication at Purdue University. Her research focuses on organizational identity and socialization, team communication, ethical reasoning development and assessment, and innovation and design. Megan holds a B.A. in communication from Saint Louis University and a M.A. in Organizational Communication from the University of Cincinnati.

\section{Prof. Patrice Marie Buzzanell, Purdue University, West Lafayette}

Patrice M. Buzzanell is a Professor in the Brian Lamb School of Communication and the School of Engineering Education (courtesy) at Purdue University. Editor of three books and author of over 140 articles and chapters, her research centers on the intersections of career, gender, and communication, particularly in STEM. Her research has appeared in such journals as Human Relations, Communication Monographs, Management Communication Quarterly, Communication Theory, Human Communication Research, and Journal of Applied Communication Research, as well as proceedings for ASEE and FIE. A fellow and past president of the International Communication Association, she has received numerous awards for her research, teaching/mentoring, and engagement. She is working on Purdue-ADVANCE initiatives for institutional change, the Transforming Lives Building Global Communities (TLBGC) team in Ghana through EPICS, and individual engineering ethical development and team ethical climate scales through NSF funding as Co-PI. [Email: buzzanel@purdue.edu]

\section{Dr. William C. Oakes, Purdue University, West Lafayette}

William (Bill) Oakes is the Director of the EPICS Program and Professor at Purdue University. He is one of the founding faculty members in the School of Engineering Education with courtesy appointments in Mechanical, Environmental and Ecological Engineering as well as Curriculum and Instruction in the College of Education. He has received numerous awards for his efforts at Purdue including being elected as a fellow of the Teaching Academy and listed in the Book of Great Teachers. He was the first engineer to receive the U.S. Campus Compact Thomas Ehrlich Faculty Award for Service-Learning. He was a co-recipient of the U.S. National Academy of Engineering's Bernard Gordon Prize for Innovation in Engineering and Technology Education and the recipient of the ASEE Chester Carlson Award for Innovation in Engineering Education. He is a fellow of ASEE and the National Society of Professional Engineers (NSPE). 


\section{Dr. Alan D. Mead, Illinois Institute of Technology}

Alan D. Mead, Ph.D., is an Assistant Professor of Psychology at the Illinois Institute of Technology where he teaches individual differences, psychometrics, structural equations modeling, meta-analysis, research methods, and statistical analysis. He sits on the editorial board for Journal of Business and Psychology and the Journal of Computerized Adaptive Testing. Since 1989, he has published over 80 peer-reviewed articles, book chapters and conference presentations. Prior to joining the faculty at IIT, he spent several years as a consultant, research scientist, and psychometrician. Dr. Mead received his Ph.D. in psychology from University of Illinois-Urbana in 2000 with a concentration on I/O psychology and a minor concentration on quantitative psychology. 


\title{
The Development of an Instrument for Assessing Individual Ethical Decision-making in Project-based Design Teams: Integrating Quantitative and Qualitative Methods
}

\begin{abstract}
Facilitating the development of ethical reasoning in engineering students is an important part of engineering education and the accreditation criteria of ABET. Project-based design has become a prominent pedagogy within current engineering education and offers opportunities where ethical considerations concerning technology, society, people, and the environment often arise. Engineering ethicists in project-based design experiences have increasingly realized the significance of introducing ethical decision-making to engineering students. However, a major challenge is the lack of effective tools for assessing students' ethical decision-making strategies, skills, and developmental processes in project-based design teams.

This paper contributes to both ethical reasoning development in engineering and project-based design through the development of an instrument, the Engineering Ethical Reasoning Instrument (EERI), for assessing individual ethical decision-making in a project-based design context. First, we present the ethical theoretical framework (e.g., Kohlberg's moral development theory) and practical background (e.g., micro and macro ethics in engineering) for the development of the instrument. In this paper, we first present the ethical theoretical framework (e.g., Kohlberg's moral development theory) and practical background (e.g., micro and macro ethics in engineering) for the development of the instrument. Second, we describe the process in which the EERI has been developed, including the validation processes which included psychometric analysis, expert review, and qualitative methods including non-participatory observations and semi-structured interviews for triangulation. Finally, we discuss some limitations or conditions of our instrument and propose suggestions for further research with the aim of improving the practical effectiveness of the EERI in assessing students' individual ethical decision-making in project-based design environment.
\end{abstract}

Keywords: Ethical decision-making; Moral development; Ethical assessment; Mixed methods.

\section{Individual Ethical Reasoning in Project-based Design Context}

Ethics and the development of ethical reasoning in engineering students is an important part of engineering education and the accreditation criteria of ABET. ABET's engineering accreditation criteria specify that graduates in accredited engineering programs are expected to acquire "an ability to design a system, component, or process to meet desired needs with realistic constraints as economic, environmental, social, political, health and safety, manufacturability, and sustainability" and "an understanding of professional and ethical responsibility". In the United States, engineering ethics education has primarily relied on three "ethical resources": codes of ethics, ethical case studies, and ethical theories. ${ }^{2}$ Teaching "abstract" codes of ethics and ethical theories is necessary but not sufficient for improving students' abilities to incorporate ethical considerations in engineering practice, as engineering practice often involves a variety of "particular" and situational moral judgments. A problem with current case pedagogy is that it is often focused on individualistic (interpersonal relations between engineers and managers and other stakeholders) and preventative (engineers are ought not to do something) themes and 
neglects the nature of everyday engineering practice (e.g., design, project/team-based, "problemsolving"). ${ }^{3}$ How to assess and improve students' ethical reasoning and decision-making skills in project-based design context has not been paid enough attention.

Within current engineering education, project-based design has become a more prominent pedagogy and offers opportunities where ethical considerations concerning technology, society, people and the environment often arise. Engineering ethicists in project-based design experiences have increasingly realized the significance of introducing ethical decision-making to engineering students. However, a major challenge is the lack of effective tools for assessing students' ethical decision-making strategies, skills, and developmental processes in project-based design teams. A few moral psychologists and philosophers have started using quantitative methods to develop measuring instruments and assess moral development of students in science and engineering programs. However, most of their experimental research and instruments are focused on the responsible conduct of research (RCR) instead of ethical decision-making in solving practical scientific and engineering problems. One of the successful instruments developed for evaluating students' ethical reasoning in the RCR is the Engineering and Science Issues Test (ESIT) developed by researchers at Georgia Institute of Technology.

This paper describes the development of an instrument, the Engineering Ethical Reasoning Instrument (EERI), for assessing individual ethical decision-making in a project-based design context. This instrument is part of a larger research project which seeks to understand the relationship between individual moral reasoning and team ethical climate in multi-disciplinary student project teams.

In this paper, we first present the ethical theoretical framework (e.g., Kohlberg's moral development theory) and practical background (e.g., micro and macro ethics in engineering) for the development of the instrument. Second, we describe the process and procedures through which the EERI has been developed. Taking inspiration from the successful Defining Issues Test, Version 2 (DIT2), we tailored ethical reasoning phases to the specific context of project-based engineering design. For example, the EERI consists of design ethics scenarios that require students to make situational ethical judgments and then rate and rank a series of items that they think are crucial for decision-making. We describe the validation processes of the EERI by means of statistical validation, expert review, and qualitative methods including nonparticipatory observations and semi-structured interviews for triangulation. Finally, we discuss some limitations or conditions of our instrument and propose suggestions for further research with the aim of improving the practical effectiveness of the instrument in assessing students' individual ethical decision-making in project-based design environment.

\section{Theoretical Framework and Practical Background}

\section{Kohlberg's Moral Development Theory}

One of the fundamental theories inspiring and supporting the development of our instrument is Lawrence Kohlberg's moral development theory. Kohlberg's moral development theory has been persistently and widely used to understand and assess moral reasoning in a variety of professional fields (e.g., science, engineering, medicine, and business) across cultures. From the perspective of moral cognition, Kohlberg's theory attempts to understand how people reason morally and on what values their reasoning processes are based. 
Kohlberg divides moral development into three levels and each level consists of two stages. The first level, preconventional level, is concerned with a predominantly self-interested orientation. Individuals reasoning in the preconventional phase conduct their ethical reasoning on the basis of "personal feelings" or self-interests. The second level, conventional level, is based on concerns for external factors (other people and authoritative rules/orders). Individuals reasoning in the conventional phase make ethical decisions based on their concerns about values related to others (some people) not oneself (e.g., values expressed in friendship, loyalty, social approval, authority, and social rules). The third level, postconventional level, builds ethical reasoning on universal norms and values (e.g., justice, human rights) that are concerned with and good for everyone in the world. Individuals operating at postconventional phases hold a critical and reflective stance on moral values and "authoritative" principles. Moral values and principles are not unquestionably accepted but subject to critique and reflection. Those who reason at this level have the highest level of moral development compared to people at the two earlier levels.

As early as the late 1970s, Kohlberg's theory was applied by engineering ethicists in assessing the moral development of professional engineers. Most typically, Richard McCuen suggested six categories of professional engineering morality corresponding to Kohlberg's six stages of moral development $^{4,5}$. Table 1 compares Kohlberg's moral development theory with McCuen's professional moral theory.

\section{Table 1. The Comparison of Kohlberg's Moral Development Theory and McCuen's Professional Moral Theory}

\begin{tabular}{|l|l|l|l|}
\hline Kohlberg's Moral Development Theory & McCuen's Professional Moral Theory \\
\hline Preconventional & Stage 1: Obedience & Preprofessional & $\begin{array}{l}\text { Stage 1: not concerned with } \\
\text { professional responsibilities; } \\
\text { "the gain for the individual" }\end{array}$ \\
& $\begin{array}{l}\text { Stage 2: Purposeful } \\
\text { exchange }\end{array}$ & & $\begin{array}{l}\text { Stage 2: "the motive of self- } \\
\text { advancement" }\end{array}$ \\
\hline Conventional & $\begin{array}{l}\text { Stage 3: Being a nice } \\
\text { person }\end{array}$ & \multirow{2}{*}{ Professional } & Stage 3: loyalty to the firm \\
\cline { 2 - 2 } & Stage 4: Law and order & & $\begin{array}{l}\text { Stage 4: loyalty to the } \\
\text { profession }\end{array}$ \\
\hline Postconventional & Stage 5: Societal consensus & $\begin{array}{l}\text { Principled } \\
\text { Professional }\end{array}$ & $\begin{array}{l}\text { Stage 5: "service to human } \\
\text { welfare is paramount" }\end{array}$ \\
\cline { 2 - 2 } & $\begin{array}{l}\text { Stage 6: Recognition and } \\
\text { acceptance of universal } \\
\text { moral principles }\end{array}$ & & $\begin{array}{l}\text { Stage 6: "professional conduct } \\
\text { follows rules of universal } \\
\text { justice, fairness, and caring for } \\
\text { fellow humans." }\end{array}$ \\
\hline
\end{tabular}

As an influential and widely used theory, Kohlberg's theory has also received criticisms from scholars with distinct disciplines. In this sense, neo-Kohlbergian theorists made various changes to classical Kohlberg's framework due to different purposes of theoretical and practical inquiries. Related to our research, in the next section, we will briefly introduce what changes have been made to Kohlberg's model by neo-Kohlbergian theorists to measure individual ethical reasoning development. 


\section{Micro Ethics and Macro Ethics in Engineering}

Engineering ethicist Joseph Herkert distinguishes two kinds of approaches to ethical practice in engineering: micro ethics and macro ethics. According to Herkert, micro ethics is concerned with engineers as individuals and the "internal relations of the engineering profession." In contrast, macro ethics refers to "the collective social responsibility of the engineering profession and the societal decisions about technology."6

For professional engineers, ethical decision-making in their everyday practice involves not only relational moral reasoning (e.g., ethical relationships between engineers and managers and other stakeholders in engineering community) but also technical decisions that have broader ethical impacts in society. The relational moral reasoning often is guided strictly by authority or ethical codes and related to "some people" in society who are actors/stakeholders and closely associated in engineering practice. In contrast, sociotechnical decision-making is often concerned with the interests of the majority of the public (sometimes all human beings). Hence, individual ethical reasoning in engineering profession often covers both conventional/professional and postconventional/principled professional levels. As taught in traditional engineering ethics classes, ethical reasoning instructed simply focuses on the conventional/professional level. Moreover, in the project-based design context, ethical reasoning includes both micro ethical reasoning (what are ethical relationships between engineers and managers, clients, and other stakeholders) and macro ethical reasoning (how technical designs/decisions affect society). Given demands for ways to teach ethical reasoning beyond traditional emphases and to account for project-based design contexts, an instrument that could address these complexities was required.

\section{EERI Development Process}

The most widely used and successful measuring instrument base on Kohlberg's theory is the DIT2 (Defining Issues Test, Version 2). As pointed earlier, in order to make Kohlberg's theory more suitable for developing measuring instrument and assessing ethical reasoning, neoKohlbergian theorists have made some changes to Kohlberg's original theoretical framework. A major revision made by the developers of the DIT2 is that the DIT2 has reworked Kohlberg's moral development phases ("stages") into schema. Rest, Narvaez, Beneau, and Thoma argue for a different view of Kohlberg's stages that moral development is seen as "shifting contributions" rather than "the staircase metaphor." ${ }^{\text {" }} \mathrm{We}$ argue that this revision was fundamental for developing engineering ethical reasoning instrument based on the DIT2 framework because engineers normally have multiple ways of thinking about most phenomena and ideally "the more primitive ways of thinking are gradually replaced by more advanced ways of thinking" 7 .

However, a limitation with the DIT2 is that it has mainly been used to assess general moral development rather than the peculiarities of ethical situations in engineering practice. Although our instrument development process was inspired by the successful structure of the DIT2, we tailored our engineering ethical reasoning instrument to the specific context of project-based engineering design. The EERI consists of design scenarios that require students to make situational ethical judgments and then rate and rank a series of items that they think are crucial for decision-making.

Like the DIT2, the EERI uses three fundamental schemas based on Kohlberg's moral development phases: preonventional (focusing on personal interest and encompassing 
Kohlberg's stages 2 and 3), conventional (maintaining norms, equivalent to Kohlberg's stage 4), and postconventional (perspective-taking, ability to appeal to ideals that are shareable and nonexclusive, and expectations for full reciprocity between laws and the individual, Kohlberg's stages 5 and 6). ${ }^{8}$ The instrument has been developed at Purdue University over several years in collaboration with Illinois Institute of Technology, Lehigh University, and Michigan Technological University. Eight scenarios were developed: Housing Quality, Soap Box Derby, International Aid, Flood Control, Nurse Schedule Software, Water Quality, Grant Proposal, and Pedestrian Bridge. These scenarios are adapted from actual student projects and involve engineering contexts such as issues of safety, design standards, and constraints of cultural norms.

In these scenarios, students are situated in ethical decision-making environments and given ethical dilemmas that are very close to the issues what they may encounter in their design projects. Different from Kohlberg's approach using an interview that asks participants to solve moral dilemmas and explain their choices, ${ }^{9}$ our instrument instructs students to indicate explicitly what action they would like to take in the given situations. In the following example, the scenario "Soap Box Derby" is presented with the follow-up question, "would you lie to the child?", requiring a specific ethical decision by research participants:

Your student design team has designed a new Soap Box Derby car that allows children with physical and cognitive disabilities to race by allowing an adult to ride in a backseat and maintain full control of the car. Based on suggestions from the adults, you have added spring tension to the child's steering wheel in front in order to simulate the feeling of driving and make the child's experience more realistic and fun. The child will not have the ability to control the car, only the illusion of control. Before the first test run with an adult and a 14-year-old child on board you hear the child's parent tell the child to "be careful" and to "drive safety." The parent turns to you, explains that because of a cognitive disability the child likely won't understand the difference anyway, and asks you to tell the child that the front steering wheel is actually functional. The request that you lie to the child would take advantage of the child's disability and it creates the possibility that the child would feel responsible if they were to lose the race or have an accident. Would you lie to the child? _Yes__Can't decide__No

After indicating the decisions they are likely to make in this scenario, students are asked to rate the importance of a series of items in making their decisions. Students rate a number of items in terms of importance with five different response alternatives: great, much, some, little, or no.

Example statements include:

- Are you uncomfortable disagreeing with the parent?

- Are there rules against lying in this case?

- Does the potential benefit outweigh the potential downside?

- Whether your professional code of ethics requires you to "avoid deceptive acts."

- Would the parent's opinion of you be affected by your behavior?

- Can lying serve the purpose of good?

- Is it inappropriate to interfere with the parent/child relationship?

Finally, students are asked to rank the top 4 most important items from among the items they just rated.

\section{Scoring}


Based on students' rating and ranking data, quantitative analyses are conducted to ascertain students' phases in individual engineering ethical reasoning. There are two most important scores in the quantitative results:

- P score: the degree to which postconventional thinking is prevalent;

- $\quad$ N2 score: the degree to which postconventional thinking is present and preconventional thinking is absent.

Both scores are generated according to the guidelines outlined for the DIT2 ${ }^{10}$. Higher P scores are obtained by ranking the postconventional items as important and a maximum score is achieved when only the postconventional items are ranked as important. The ratings data are not used by the P score, but the $\mathrm{N} 2$ score combines the $\mathrm{P}$ score with a measure of differentiation of the ratings for pre- and postconventional items.

\section{EERI Validation Processes}

In order to validate the instrument, we used a "mixed" approach involving both quantitative (statistical validation) and qualitative methods (e.g. expert review, non-participatory observation, semi-structured interview, focus group).

Version 1 of the EERI had the eight original scenarios. This version had short instructions that did not include an example for the participants to complete. Each scenario had 12 items. Version 1 was administered to 312 participants. Engineering experts who are members of our advisory panel on our project reviewed the scenarios, items, and preliminary results. In addition, we recruited an analyst group consisting of $15 \mathrm{Ph}$.D. research students from English and Philosophy Departments to review language used in writing scenarios and statements. Based on the feedback of both review groups and the psychometric analysis of the pilot data, we made changes to the instructions, the scenarios, and the items. For example, a rating statement might have been reworded to make it clearer. We included additional items for each scenario so we could see how the items performed, with the plan to reduce the number of items using the pilot data. As a result, the scenarios of Version 2 of the instrument had $16-24$ items each which included additional items for each schema that is required for scoring. It was administered to 175 participants.

Results from the Version 2 pilot identified several items that did not perform as well as or similarly to other items in the same schema. In Version 3, these items were eliminated if the schema was already represented adequately or revised if the item was needed to represent the schema. For example, the conventional item "Are some of these buildings in violation of the city's building code?" was changed to "Shouldn't the city's building codes be applied consistently?" so it was not a question of fact, but a question of value. In addition, the personal interest item "What if you could be held responsible for negative outcomes?" was changed to "What if you could be blamed for negative outcomes?" because the original statement was chosen with frequencies similar to post-conventional items rather than self-interest. We hypothesized that the word "blamed" would better engage self-interest schemas, whereas the word "responsible" appeared to be engaging higher order reasoning. In addition, we eliminated two scenarios on the basis of their slightly lower internal consistency (participants ratings were less consistent), slightly less compelling content, and because these two scenarios were more redundant with the remaining scenarios. 
Version 3 has been administered to over 800 participants. The main psychometric analyses completed to date are coefficient alpha reliabilities. These indexes measure the degree to which responses are internally consistent and estimate the psychometric reliability (i.e., accuracy and short-term repeatability) of the scores. As a loose heuristic, reliability estimates of at least 0.70 are often required. The estimated reliability of the ratings across the six scored scenarios ranges from 0.72 to 0.85 except for one scenario (scenario 5) which as an estimated reliability of 0.565.

In order to validate both the instruments and the model of the relationship between individual moral reasoning and team ethical climate, we conducted semi-structured interviews and nonparticipatory observations with selected participants at the four engineering programs at the four collaborative universities. In total, we conducted 51 interviews and numerous observations within the four engineering programs. Interviews were, on average 40 minutes (range of 20-60 minutes), with 7 to 15 interviews conducted at each university based on student availability and desire to volunteer. The interviews were audiorecorded and later transcribed for analysis. Participants provided informed consent and were compensated for their time.

The interview protocol was designed to engage concepts incorporated related to both the individual and team climate instruments we are developing, but also included questions that elicit information about how participants prioritize issues related to their individual decision-making. We also explore general team processes and the students' perceived roles within their teams to provide context on how these individuals operate within their teams. The interview protocol was semi-structured such that similar guiding questions were asked of most participants with secondary questions designed to probe participants' responses. The primary guiding questions were: ${ }^{11}$

- How would you characterize your team interactions as a whole?

- What is important to or valued by your team? What are your team's priorities?

- What is your role on the team? Do you feel like you belong? Are your viewpoints listened to?

- How and when are decisions made by your team? Who was involved in those decisions?

- Do you feel as though any of these decisions or your team work involved ethical considerations?

- How do you define ethics? How do you make ethical decisions?

- Does your team seem concerned about professional codes and/or rules/laws?

- Does your team share a common understanding of "right and wrong"?

We conducted observations of team meetings to provide context for the interviewers and help identify participants for the interviews. Observations were guided by a general observation protocol, with other interesting interactions being noted where appropriate. Several examples of the observation protocol include:

- What seems most important to the team at this time? What did you observe that made you answer in the way you did?

- How were decisions made during team meetings? What were the decisions about? Who made the decisions?

- Did you observe interactions or language centered on how a decision or design aspect might affect individual team members? 
- Did you observe interactions or language that centered on how team processes and deliverables align with moral or ethical stances that are up for discussion, shared and malleable, and/or act as appeals to ideals for human existence?

Based on the discourse analysis and thematic analysis of data from semi-structured interviews and non-participatory observations, we were able to relate qualitative findings to quantitative results (e.g., P score and N2 score). Through use of mixed methods, we were able to delve into how interviewees perceived and reported their team processes, particularly their individual and team ethical considerations, within their project-based design projects. We related interview and observational data to individual quantitative scores from certain ethical schemas as well as whether and how team climate seemed to impact individual ethical reasoning. Analysis of the interview and observational data revealed evidence of the three different stages of moral development probed in the EERI.

The use of interviews and observations gave us access to unanticipated aspects of individual ethical decision making and moral reasoning by allowing participants to go into greater detail about, and reflect on, the concepts underlying the instruments. Participants offered insights into aspects of ethical decision making that were not directly present in the instrument, as well as offering a richer description of the often complex team interactions as the participants experienced them.

\section{Limitations, Conditions and Future Work}

In conclusion, we discuss some limitations or conditions of our instrument and propose suggestions for further research with the aim of improving the practical effectiveness of the instrument in assessing students' individual ethical decision-making in project-based design environment.

\section{Alternative Theoretical Framework(s)}

One of the most important issues we might want to take into account in our future research is: to what extent do Kohlbergian-based instruments, and specifically the EERI, account for the different kinds of ethical reasoning required by engineers in their design processes? Are there alternative theoretical bases that better account for the ethical considerations faced by engineers during design processes (e.g., Confucian "role ethics" may bring insight views on communitarian aspects of individual ethical reasoning in interactive and relational team context.)? If so, how might these different ways of understanding and measuring ethical decision-making help us improve our current instrument for individual ethical reasoning or build upon this base for more complex measurements?

\section{Individual Differences}

Another issue we might want to consider in future research is to evaluate if and how individual differences (e.g., ethnicity, gender, cultural background, social class) contribute to individual ethical reasoning in engineering design contexts. These differences may or may not contribute to formulate different ethical reasoning in practice in project-based team contexts. Research in this direction may help us better understand the relationships between the three different schemas. For instance, one may ask why some engineers prioritize considerations of universal values (e.g. fairness, human rights, social welfare) over conventional considerations while some engineers do 
not. Although the neo-Kohlbergian approach may see this prioritization as a

developmental/cognitive achievement, we question that general assessment. Specifically, engineering requires and complex dialectical interplay between conventional concerns such as safety and adherence to normative procedures that otherwise might jeopardize design integrity and relationships among stakeholders and postconventional ethical thinking that questions design processes by looking for unintended and, perhaps, ironic ethical consequences for people and the construction and use of artifacts. These considerations may align with reflections by moral psychologists who have pointed out that conservatives (those who do not prioritize) may have the more complex moral system. ${ }^{12}$ Examining whether and how individual differences contribute to the complexities and nuances of ethical reasoning in engineering practice is an essential step in further developments of individual and team ethical processes.

\section{Multicultural Context}

One of the critiques of Kohlberg's moral development theory is that it tends to assume a Western democratic context of ethical decision-making. As the international population among engineering students continues to increase dramatically, engineering educators need to rethink broadly what other subcategories may be included in the three moral schemas to account for cross-cultural and global contexts. For instance, what do conventional and postconventional thinking mean in a broader and cross-cultual/multicultural context? Such considerations may help us to redesign scenarios and statements to make the instrument more inclusive and explanatorily effective for multicultural and international contexts.

\section{Acknowledgements}

We would like to acknowledge the work of Craig Titus in developing the EERI, as well as our collaborators at our partner institutions: Kemp Ellington, Jill May, and Danny Gandara at Illinois Institute of Technology; Mary Raber at Michigan Technological University, and John Ochs and Lisa Getzler-Linn at Lehigh University. This work was made possible by a grant from the National Science Foundation (DUE-112374). Any opinions, findings, and conclusions or recommendations expressed in this material are those of the authors and do not necessarily reflect the views of the National Science Foundation.

\section{References}

[1] ABET. (2013). Criteria for Accrediting Engineering Programs, 2012 - 2013. Available at: http://www.abet.org/DisplayTemplates/DocsHandbook.aspx?id=3143.

[2] Herkert, J. (2000). Engineering ethics education in the USA: Content, pedagogy, and curriculum. European Journal of Engineering Education, 25, 303-313.

[3] Bucciarelli, L. Ethics and engineering education. European Journal of Engineering Education, 33(2), $141-149$.

[4] McCuen, R. (1979). The ethical dimensions of professionalism. Journal of Professional Activities, 105 (12), $89-$ 105. 
[5] Vesilind, P., \& Gunn, A. (1998). Engineering, ethics, and the environment. New York, NY: Cambridge University Press.

[6] Herkert, J. (2001). Future directions in engineering ethics research: Microethics, macroethics and the role of professional societies. Science and Engineering Ethics, 7 (3), 403-414.

[7] Rest, J., Narvaez, D., Bebeau, M., \& Thoma, S. (1999). A neo-Kohlbergian approach: The DIT and schema theory. Educational Psychology Review, 11 (4), 291-324.

[8] Zoltowski, C., Buzzanell, P., Oakes, W. (2013). Utilizing an engineering ethical reasoning instrument in the curriculum. Paper presented at the 120th ASEE Annual Conference \& Exposition, Atlanta, GA.

[9] Rest, J., Narvaez, D., Bebeau, M., \& Thoma, S. (1999). A neo-Kohlbergian approach: The DIT and schema theory. Educational Psychology Review, 11 (4), 291-324.

[10] Rest, J., Thoma, S., Narvaez, D., \& Bebeau, M. (1997). Alchemy and beyond: Indexing the defining issues test. Journal of Educational Psychology, 89 (3), 498-507.

[11] Zoltowski, C., Buzzanell, P., Oakes, W., \& Kenny, M. (2013). A qualitative study exploring students' engineering ethical reflections and their use in instrument validation. Paper presented at the 2013 IEEE Frontiers in Education Conference, Oklahoma City, OK.

[12] Baril, G., \& Wright, J. Different types of moral cognition: Moral stages versus moral foundations. Personality and Individual Differences, 53 (4), 468-473. 